\title{
A Case of Dapsone Syndrome: A Case Report
}

\author{
Dr. Vishakha Sinha V ${ }^{1}$, Dr. Akhil Giradkar B $^{1}$, Dr. Kalikar Mrunalini Vinay ${ }^{2}$, Dr. Dakhale Ganesh Nathuji ${ }^{3}$ \\ 1. Junior Resident in Pharmacology, 2. Associate Professor in Pharmacology, 3. Professor in Pharmacology, \\ Govt. Medical College, Nagpur, M.S. 440003, India. \\ *Corresponding author's E-mail: mrunalinikalikar@yahoo.com
}

Received: 12-06-2020; Revised: 19-08-2020; Accepted: 27-08-2020.

DOI: 10.47583/ijpsrr.2020.v64i01.014

\begin{abstract}
Dapsone used for leprosy and also for several inflammatory conditions can be sometimes fatal. It is generally well tolerated but can also cause adverse drug reactions (ADR) in some patients. One of the less common ADR is hypersensitivity reactions known as dapsone syndrome. It is generally characterized as a combination of at least two of the following four symptoms: fever, lymph adenopathy, generalized rash and hepatitis occurring after dapsone intake. The complete syndrome consists of all four symptoms. We report a 26-year-old lady on multidrug therapy treatment for lepromatous leprosy for past one month presented with the history of yellowish discoloration of skin and sclera which was associated with intense itching for 8 days who was prescribed dapsone. This report will caution clinicians about this side effect of dapsone which is commonly prescribed.
\end{abstract}

Keywords: Leprosy, Dapsone, Adverse drug reaction.

\section{INTRODUCTION}

T he history of dapsone as an oral drug dates back to 1949. Initially it was primarily used for leprosy for which it is still used, but in addition to that it acts as an anti-inflammatory agent in many other inflammatory dermatological conditions. ${ }^{1}$ Dapsone hypersensitivity reaction was known to man since 1950 and was reported by Lowe. Hypersensitivity to dapsone is deemed to be the main cause of this syndrome. ${ }^{2}$ In general, dapsone has good tolerability and is suitable for long term treatment, but still adverse drug reactions (ADR) may occur. Haemolytic anaemia and methemoglobinemia are the known and frequent dose dependent adverse effects. Major, less known, potentially fatal rare ADRs with unknown pathomechanisms include hypersensitivity reactions (HR) to dapsone like dapsone syndrome. ${ }^{1,3}$ One such case lepromatous leprosy who developed dapsone hypersensitivity syndrome is reported.

\section{CASE REPORT}

A 26-year-old female who was on multidrug therapy treatment for lepromatous leprosy for past one month presented with the history of yellowish discoloration of skin and sclera which was associated with intense itching for 8 days. On admission she was afebrile, normotensive. She had pallor and pruritic maculopapular exanthematous rash on extremities and face. Other system examinations were unremarkable.

Investigations revealed hemoglobin $5.8 \mathrm{gm} \%$, white blood cells $0.8 / \mathrm{mm} 3$, platelets $56000 / \mathrm{mm} 3$, serum bilirubin 13.5 . Viral hepatitis B surface antigen, hepatitis $E$ and hepatitis C were negative. The levels of urea, creatinine, uric acid and serum electrolytes were normal. Her HIV screening, blood culture, urine culture was negative. Abdominal ultrasound showed liver to be normal, gall bladder was well distended, there was no evidence of portal hypertension or biliary obstruction. Her chest radiograph was normal.

The symptoms hepatotoxicity (also evident by raised bilirubin levels) occurred in less than 8 weeks after starting dapsone therapy, was not attributable to any other drug which was used concurrently and also the symptoms was unrelated to leprosy or any other underlying disease. So, a diagnosis of DHS (Dapsone hypersensitivity syndrome) was made.

Thus, dapsone was stopped and corticosteroids were given both orally and parenterally. She was also given blood transfusion as her hemoglobin were low. Patient's clinical condition improved after two weeks and laboratory tests were also around normal. Her blood counts returned to normal. The steroids were slowly tapered. Patient was discharged after her blood investigations became normal and her serum bilirubin decreased to 1.8. On discharge she was continued on tablets Clofazimine and Rifampicin as alternative drug after stopping dapsone. For corticosteroids, tablet Prednisolone was initially given at the dose of $40 \mathrm{mg}$ then at discharge it was tapered to 30 $\mathrm{mg}$.

\section{DISCUSSION}

Dapsone syndrome occurs five to six weeks after the intake of dapsone and so sometimes also called as 5 weeks dermatitis. This syndrome also known by the name of "sulfone syndrome" consists of exfoliative dermatitis and or various cutaneous manifestations, lymphadenopathy, hepato-splenomegaly, mononucleosis, fever, jaundice and hepatitis. The cutaneous lesions are almost always present but the presence of the other features may not be there in all cases. ${ }^{3,4}$ According to Richardus and Smith, a true 
diagnosis of Dapsone Hypersensitivity Syndrome should have following criteria:

a) Symptoms should occur in less than 8 weeks after starting dapsone therapy and should resolve after withdrawing the drug.

b) Symptoms should not be attributable to any other drug used concurrently.

c) Symptoms should be unrelated to leprosy or any other underlying disease.

In this case the patient had pancytopenia and low haemoglobin which was suggestive of bone marrow suppression and haemolysis due to dapsone. Hyperbilirubinemia seen in dapsone syndrome also occur due to haemolysis in addition to hepatotoxicity. Hepatocellular injury in dapsone syndrome is evident by elevated transaminases, and liver biopsy in such cases may show predominantly eosinophilic lobular and portal infiltration. Hepatitis may progress to hepatic failure and can be fatal. ${ }^{4}$ Cholestatic pattern of injury may have less severe course and high alkaline phosphatase level and modest rise in transaminases level is seen. Granulomas may be seen in liver biopsy.

Most patients recovered after dapsone withdrawal, and in few cases with glucocorticosteroids treatment, however approximately $10.2 \%$ had a fatal outcome. Most common cause of death being the hepatic come. Discontinuation of dapsone therapy as early as possible improves the prognosis. It seems likely that the hypersensitivity syndrome to dapsone is a dose-independent drug reaction. Relative higher risk for development of hypersensitivity syndrome is present is present in first 5 months of treatment. During this period, frequent clinical and laboratory evaluations are important and may help in early prediction and diagnosis. However, there is no reliable way to predict the risk for

dapsone hypersensitivity. If suspected, a prompt discontinuation of dapsone therapy happens to be the best way to improve prognosis . ${ }^{3}$

The hypersensitivity syndrome to dapsone must be considered an extremely rare side effect as there are very less reports of the same though the drug is being used frequently.

\section{REFERENCES}

1. Lorenz Maria, Gottfried Wozel and Jochen Schmitt, Department of Dermatology, and Institute and Policlinic for Occupational and Social Medicine, University Hospital Carl Gustav Carus, Technische Universität Dresden, Dresden, Germany Acta Derm Venereol, 92, 2012, 194-199.

2. LOWE J. Treatment of leprosy with diamino-diphenyl sulphone by mouth. Lancet. 1(6596), 1950, 145-150. doi:10.1016/s0140-6736(50)90257-1 PMID: 15401496

3. Wozel VE. Innovative use of dapsone. Dermatol Clin. 28(3), 2010, 599-610. doi: 10.1016/j.det.2010.03.014 PMID: 20510768

4. Grace M. An unusual case of dapsone syndrome. Indian Dermatol Online J, 2, 2011, 88-90. PMID: 23130233.

5. Richardus JH, Smith TC. Increased incidence in leprosy of hypersensitivity reactions to dapsone after introduction of multidrug therapy. Lepr Rev. 60, 1989, 267-73. PMID: 2491425.

Source of Support: None declared.

Conflict of Interest: None declared.

For any question relates to this article, please reach us at: editor@globalresearchonline.net

New manuscripts for publication can be submitted at: submit@globalresearchonline.net and submit_ijpsrr@rediffmail.com 